\title{
A New Class of Exact Solutions for 3D Compressible Reconnection Problem
}

\author{
T. TERASAwA ${ }^{1}$, M. Hoshino $^{2}$, and M. Fujimoto ${ }^{3}$ \\ ${ }^{1}$ Department of Earth and Planetary Physics, The University of Tokyo, Tokyo 113, Japan \\ ${ }^{2}$ The Institute of Physical and Chemical Research (Riken), Wako, Saitama 351-01, Japan \\ ${ }^{3}$ Department of Physics, Nagoya University, Nagoya 464, Japan
}

(Received January 27, 1993; Revised May 24, 1993)

\begin{abstract}
For the steady-state three-dimensional reconnection problem in a compressible plasma, we have found a new class of exact solutions, which consist of ideal-MHD regions, MHD singularities (slow shocks and rotational discontinuities), and a neutral line. This solution predicts the existence of plasma jetting parallel to the neutral line, in addition to the usual plasma jetting in the direction perpendicular to the neutral line. However, contrary to the suggestion by LAU and FINN (1990), and PRIEST and FORBES (1992) that three-dimensional reconnection regions should have velocity singularities, our solution has well-behaved velocity fields without any singularity.
\end{abstract}

\section{Introduction}

At the dayside magnetopause, magnetic reconnection controls the energy transfer process from the solar wind to the magnetosphere, when the interplanetary magnetic field has a southward component. Since the interplanetary magnetic field generally has other components, sun-earth and dawn-to-dusk components, three dimensionality is essential for the reconnection problem at the magnetopause. In the study of the magnetotail, where magnetic reconnection plays an essential role during substorm energy conversion processes, theoretical consideration has been focused to the reconnection of anti-parallel field lines. Observations show, however, occasional existence of the dawn-dusk component of the magnetic field, originating in the dawn-dusk component of the interplanetary magnetic field. This component makes the field structure of the magnetotail reconnection region three dimensional also.

Recently, it is suggested that the dynamical properties in three-dimensional reconnection regions are significantly different from those in two-dimensional reconnection regions (SCHINDLER et al., 1988; Hesse and Schindler, 1988; Greene, 1988; Wright and Berger, 1989; LAu and FinN, 1990; PRIEST and ForBes, 1992). Since exact solutions for the three-dimensional reconnection problem have not been known so far, the arguments presented by the above authors are either qualitative or based on approximate solutions. In this letter, we present an exact solution for the steady-state three-dimensional reconnection problem in compressible plasmas, in which the reconnection region consists of ideal-MHD regions, MHD singularities and a neutral line. (Since we still drop the spatial dependence in one direction, $\partial / \partial z=0$, our solution might be better called a $2 \frac{1}{2}$-dimensional solution.)

\section{Description of the New Solution}

We shall consider a reconnection process between plasmas of the same pressure $P_{1}$ and mass density $\rho_{1}$. The original magnetic fields (namely the magnetic fields before reconnection) have the same magnitude $\left(\left|B_{1}\right|=\left|B_{5}\right|\right)$ but make an oblique angle $\pi-2 \theta_{B_{1}}$. The coordinate system is 
(a)

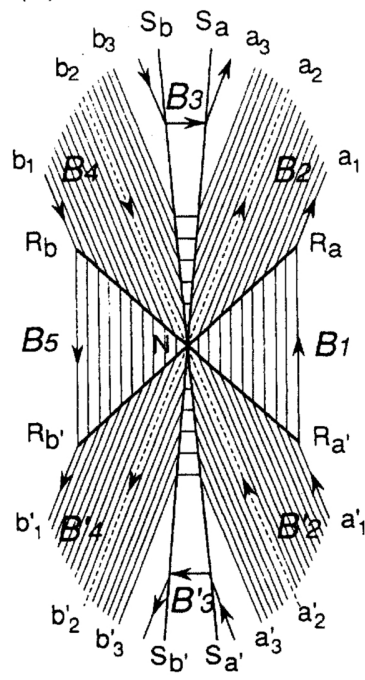

(b)

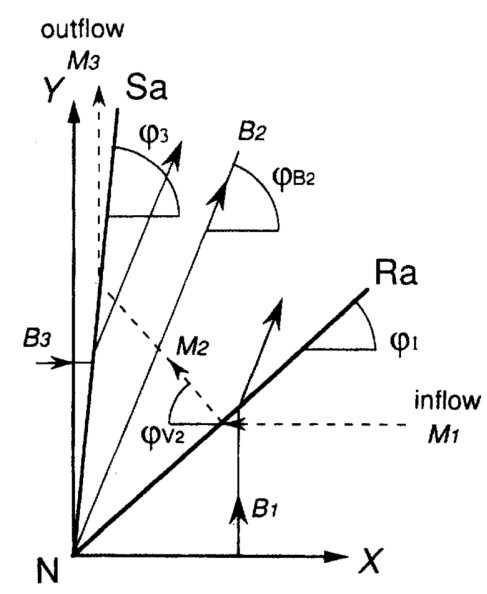

(c)

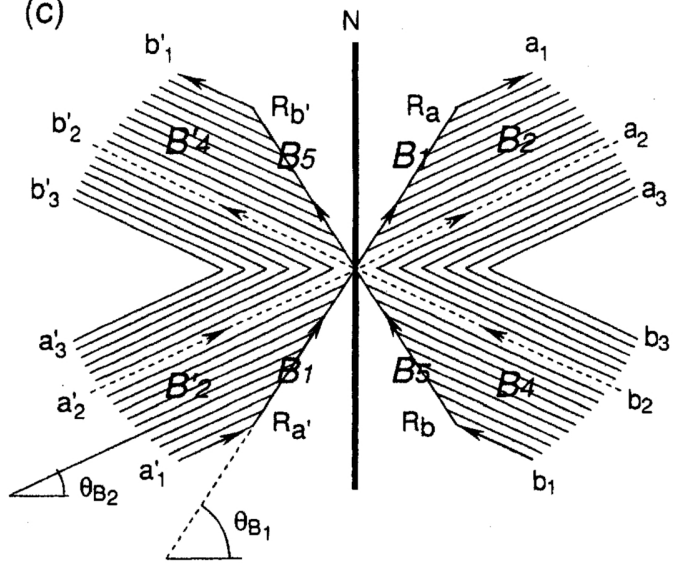

(d)

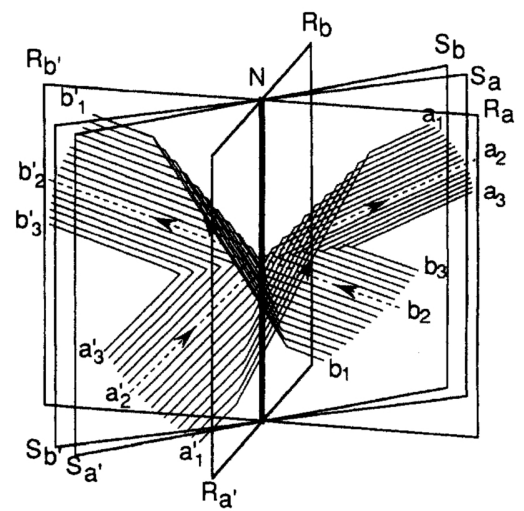

Fig. 1. Illustration of the solution for $M_{1}=0.5$ and $\beta_{1}=1$. (a) An $x y$ cross section of the reconnection region (namely a view looking into the $-z$ direction) (b) A quarter view of the panel (a). (c) An $y z$ cross section of the reconnection region (namely a view looking into the $-x$ direction) (d) A three-dimensional view of the reconnection region. Dashed lines in panels (a), (c), and (d), $\left[\mathrm{a}_{2}^{\prime} \rightarrow \mathbf{N} \rightarrow \mathrm{a}_{2}\right]$ and $\left[\mathrm{b}_{2} \rightarrow \mathbf{N} \rightarrow \mathrm{b}^{\prime}{ }_{2}\right]$, are separatrices.

taken so as to represent these fields as,

$$
\begin{aligned}
& \vec{B}_{1}=\left(0, \quad B_{1} \cos \theta_{B_{1}}, B_{1} \sin \theta_{B_{1}}\right), \quad(x>0) \\
& \vec{B}_{5}=\left(0,-B_{1} \cos \theta_{B_{1}}, B_{1} \sin \theta_{B_{1}}\right) . \quad(x<0)
\end{aligned}
$$

We require that during the reconnection process the field lines as well as the stream lines are straight except bends at shocks and discontinuities. We note that the requirement for the straightness is rather technical but not physical. Physically admissible solutions could have curved magnetic field lines and/or curved stream lines in the regions among shocks/discontinuities. To make the problem numerically tractable, however, we shall not consider these general solutions 
in this letter. Under the above requirement, we have found that four rotational discontinuities $\left(R_{a}, R_{b}, R_{a}^{\prime}\right.$, and $\left.R_{b}^{\prime}\right)$ and four slow shocks $\left(S_{a}, S_{b}, S_{a}^{\prime}\right.$, and $\left.S_{b}^{\prime}\right)$ are needed for the field lines to reconnect. (Without rotational discontinuities, the field lines cannot have necessary threedimensional bends. Without slow shocks the necessary energy conversion from the magnetic field to the particles cannot occur.) The geometry of our solution is illustrated in Fig. 1, where the intersection line of four discontinuities is taken as the $z$ axis. Since the $y$ component of the magnetic field is zero along this line, this is the "neutral" line $(\mathbf{N})$ as is called traditionally.

In the regions between the rotational discontinuities and slow shocks, the magnetic fields are,

$$
\begin{aligned}
& \vec{B}_{2}=\left(B_{1} \cos \theta_{B_{2}} \cos \varphi_{B_{2}}, B_{1} \cos \theta_{B_{2}} \sin \varphi_{B_{2}}, B_{1} \sin \theta_{B_{2}}\right) \text { between } \mathrm{R}_{\mathrm{a}} \text { and } \mathrm{S}_{\mathrm{a}} \\
& \vec{B}_{2}^{\prime}=\left(-B_{1} \cos \theta_{B_{2}} \cos \varphi_{B_{2}}, B_{1} \cos \theta_{B_{2}} \sin \varphi_{B_{2}}, B_{1} \sin \theta_{B_{2}}\right) \text { between } \mathrm{R}_{\mathrm{a}}^{\prime} \text { and } \mathrm{S}_{\mathrm{a}}^{\prime} \\
& \vec{B}_{4}=\left(B_{1} \cos \theta_{B_{2}} \cos \varphi_{B_{2}},-B_{1} \cos \theta_{B_{2}} \sin \varphi_{B_{2}}, B_{1} \sin \theta_{B_{2}}\right) \text { between } \mathrm{R}_{\mathrm{b}} \text { and } \mathrm{S}_{\mathrm{b}} \\
& \vec{B}_{4}^{\prime}=\left(-B_{1} \cos \theta_{B_{2}} \cos \varphi_{B_{2}},-B_{1} \cos \theta_{B_{2}} \sin \varphi_{B_{2}}, B_{1} \sin \theta_{B_{2}}\right) \text { between } \mathrm{R}_{\mathrm{b}}^{\prime} \text { and } \mathrm{S}_{\mathrm{b}}^{\prime}
\end{aligned}
$$

The magnetic fields downstream of pairs of slow shocks are,

$$
\begin{aligned}
& \vec{B}_{3}=\left(B_{3} \cos \theta_{B_{3}}, 0, B_{3} \sin \theta_{B_{3}}\right) \text { downstream of } \mathrm{S}_{\mathrm{a}} \text { and } \mathrm{S}_{\mathrm{b}}, \\
& \vec{B}_{3}^{\prime}=\left(-B_{3} \cos \theta_{B_{3}}, 0, B_{3} \sin \theta_{B_{3}}\right) \text { downstream of } \mathrm{S}_{\mathrm{a}}^{\prime} \text { and } \mathrm{S}_{\mathrm{b}}^{\prime} .
\end{aligned}
$$

For simplicity, we have required in the above that the magnetic field $\vec{B}=\left(B_{x}, B_{y}, B_{z}\right)$ and flow velocity $\vec{V}=\left(V_{x}, V_{y}, V_{z}\right)$ satisfy the following symmetry property: With respect to the $y z$ $(x=0)$ plane, $B_{x}, B_{z}$, and $V_{y}$ are symmetric, and $B_{y}, V_{x}$, and $V_{z}$ are anti-symmetric. With respect to the $x z(y=0)$ plane, $B_{y}, B_{z}$, and $V_{x}$ are symmetric, and $B_{x}, V_{y}$, and $V_{z}$ are antisymmetric. If we do not pose these requirements, a variety of asymmetric solutions results. We shall describe these asymmetric solutions in a future publication.

Figure 1a shows the $x y$ cross section of the reconnection region. A quarter view of Fig. 1a $\left(x \geq 0\right.$ and $y \geq 0$ ) is shown in Fig. $1 \mathrm{~b}$, where we define the attack angles of $\mathrm{R}_{\mathrm{a}}$ and $\mathrm{S}_{\mathrm{a}}, \varphi_{1}$ and $\varphi_{3}$. Dashed lines show the stream lines in the three regions, where flow Alfvén Mach numbers are $M_{1}\left(=\left|V_{1 x}\right| / V_{\mathrm{A} 1}\right)$ upstream of $\mathrm{R}_{\mathrm{a}}, M_{2}\left(=\left|\vec{V}_{2}\right| / V_{\mathrm{A} 1}\right)$ between $\mathrm{R}_{\mathrm{a}}$ and $\mathrm{S}_{\mathrm{a}}$, and $M_{3}\left(=V_{3 y} / V_{\mathrm{A} 1}\right)$ downstream of $\mathrm{S}_{\mathrm{a}} . V_{\mathrm{A} 1}$ is the Alfvén velocity $B_{1} / \sqrt{4 \pi \rho_{1}}$ in the upstream region of $\mathrm{R}_{\mathrm{a}}$. While the structure shown in Fig. 1a and 1b looks similar to the Sonnerup's solution (SoNNERUP, 1970), it differs in the three-dimensional sense. In particular, the coplanarity planes at four slow shocks all differ and do not coincide with the $x y$ plane. The $y z$ cross section and a three-dimensional view of the same reconnection region are shown in Fig. 1c and 1d. A field line, [ $a_{1}^{\prime} \rightarrow R_{a}^{\prime} \rightarrow R_{a} \rightarrow a_{1}$ ], is convected toward $\mathbf{N}$ in the $-x$ direction. This field line becomes a separatrix $\left[\mathbf{a}_{2}^{\prime} \rightarrow \mathbf{N} \rightarrow \mathbf{a}_{2}\right]$, when it arrives at the neutral line $\mathbf{N}$. At the neutral line, this field line is to be reconnected with another separatrix, $\left[\mathrm{b}_{2} \rightarrow \mathbf{N} \rightarrow \mathrm{b}^{\prime}{ }_{2}\right.$ ], which has been convected toward $\mathbf{N}$ in the $+x$ direction $\left.\left(\left[\mathrm{b}_{1} \rightarrow \mathrm{R}_{\mathrm{b}} \rightarrow \mathrm{R}_{\mathrm{b}}^{\prime} \rightarrow \mathrm{b}_{1}^{\prime}\right]\right] \Longrightarrow\left[\mathrm{b}_{2} \rightarrow \mathbf{N} \rightarrow \mathrm{b}_{2}^{\prime}\right]\right)$. After the reconnection, these two field lines become a pair of field lines, $\left[b_{3} \rightarrow S_{b} \rightarrow S_{a} \rightarrow a_{3}\right]$ and $\left[a^{\prime}{ }_{3} \rightarrow S_{a}^{\prime} \rightarrow S_{b}^{\prime} \rightarrow b^{\prime}{ }_{3}\right.$, ejected respectively in the $+y$ and $-y$ directions. (Note that the parts $\left[\mathrm{S}_{\mathrm{b}} \rightarrow \mathrm{S}_{\mathrm{a}}\right]$ and $\left[\mathrm{S}_{\mathrm{a}}^{\prime} \rightarrow \mathrm{S}_{\mathrm{b}}^{\prime}\right]$ in Fig. 1c, whose projections are parallel to the $z$ axis in this panel, are not well resolved graphically.)

The numerical method by which solutions are obtained is similar to that used by HAMEIRI (1979). We search a solution for a given angle $\varphi_{3}$ of the slow shock $\mathrm{S}_{\mathrm{a}}\left(0<\varphi_{3}<90^{\circ}\right)$. First, we set trial values of $\varphi_{B_{2}}$ and $\theta_{B_{2}}$. For these angles, we determine $\vec{V}_{2}$ and $\vec{V}_{3}$, the upstream and downstream velocities around $S_{a}$, using the shock Rankin-Hugoniot condition and the symmetry condition, $V_{3 x}=V_{3 z}=0$. Second, we determine the angle $\varphi_{1}$ so that the stationary condition for 
(a)
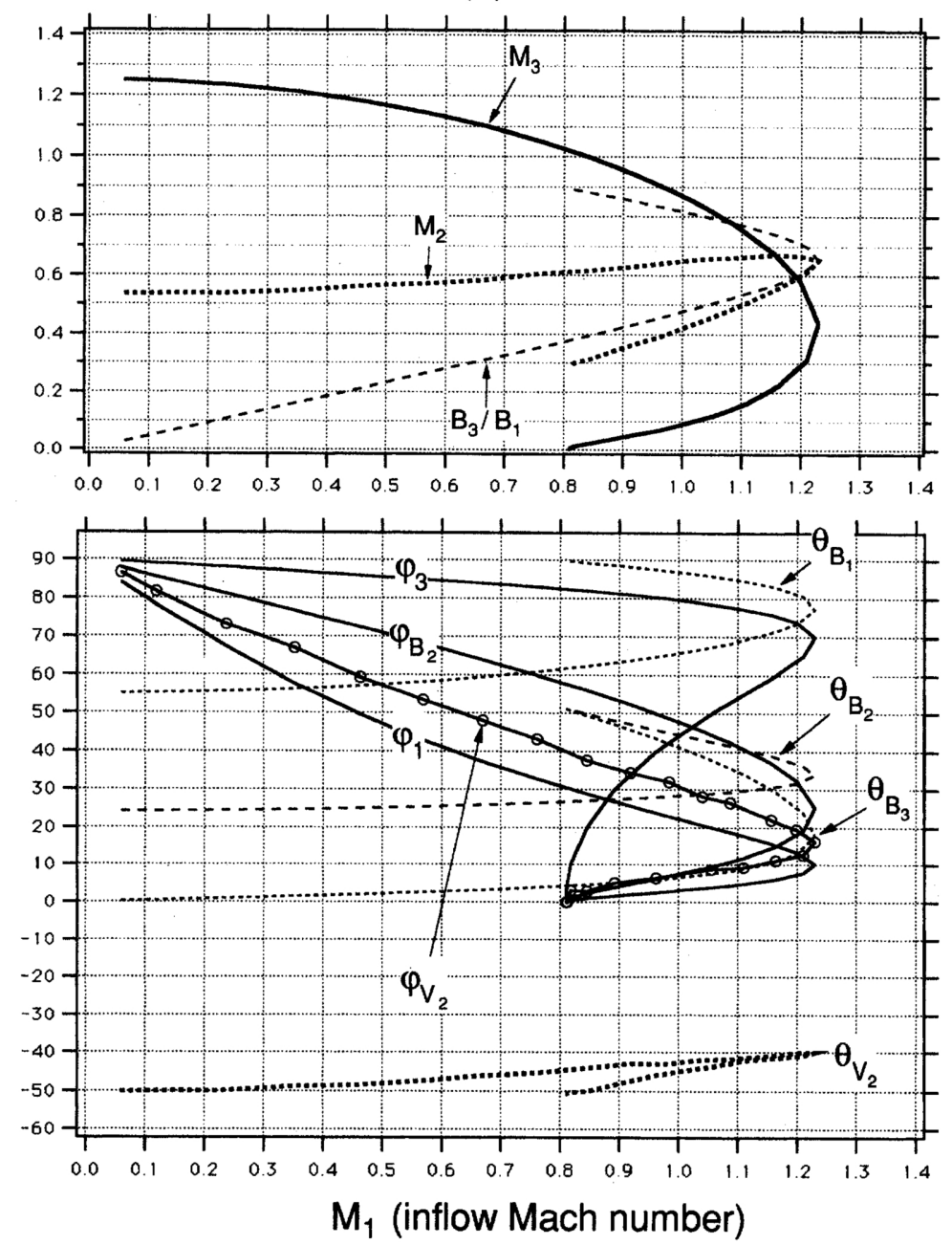

Fig. 2a. Dependence of reconnection parameters on the inflow Alfvén Mach number $M_{1}$ for the case of upstream $\beta_{1}=0$. For the angles, $\theta$ 's and $\phi$ 's, the unit of degree is used.

the rotational discontinuity $\mathrm{R}_{\mathrm{a}}, \vec{V}_{2} \cdot \vec{n}_{\text {rot }}=\vec{B}_{2} \cdot \vec{n}_{\text {rot }} / \sqrt{4 \pi \rho_{2}}$, is satisfied, where $\rho_{2}\left(=\rho_{1}\right)$ is the mass density, and $\vec{n}_{\text {rot }}$ the unit normal vector of the rotational discontinuity, $\left(-\sin \varphi_{1}, \cos \varphi_{1}, 0\right)$. Across $\mathrm{R}_{\mathrm{a}}$, the magnetic field rotates around $\vec{n}_{\text {rot }}$ as the axis, keeping its magnitude $\left(\left|\vec{B}_{2}\right|=\right.$ $\left.\left|\vec{B}_{1}\right|\right)$. Utilizing this freedom of rotation, we set $B_{1 x}=0$. Using the Walén relation for rotational discontinuities, $\vec{V}_{2}-\vec{V}_{1}=-\left(\vec{B}_{2}-\vec{B}_{1}\right) / \sqrt{4 \pi \rho_{2}}$, we determine $\vec{V}_{1}$, the velocity in the upstream region of $\mathrm{R}_{\mathrm{a}}$. By changing $\varphi_{B_{2}}$ and $\theta_{B_{2}}$ we make $\vec{V}_{1}$ point to the $-x$ direction. (The actual algorithm consists of a double bisection method searching of $\varphi_{B_{2}}$ and $\theta_{B_{2}}$ for $V_{1 y}=V_{1 z}=0$ ). Note that the theta angle of the original magnetic field, $\theta_{B_{1}}$, is not given a priori, but determined when the relation at $\mathrm{R}_{\mathrm{a}}$ is found.

The parameters $\left(M_{2}, M_{3}, B_{3} / B_{1}, \varphi_{1}, \varphi_{B_{2}}, \varphi_{3}, \theta_{B_{1}}, \theta_{B_{2}}, \theta_{B_{3}}, \varphi_{V_{2}}\right.$ and $\left.\theta_{V_{2}}\right)$ are plotted against the inflow Alfvén Mach number $M_{1}$ in Fig. $2 \mathrm{a}$ and $2 \mathrm{~b}$ for the cases of $\beta_{1}=0$ and $\beta_{1}=1$, respectively. The azimuthal angle $\varphi_{V_{2}}$ and the elevation angle $\theta_{V_{2}}$ are flow deflection angles at the 
(b)
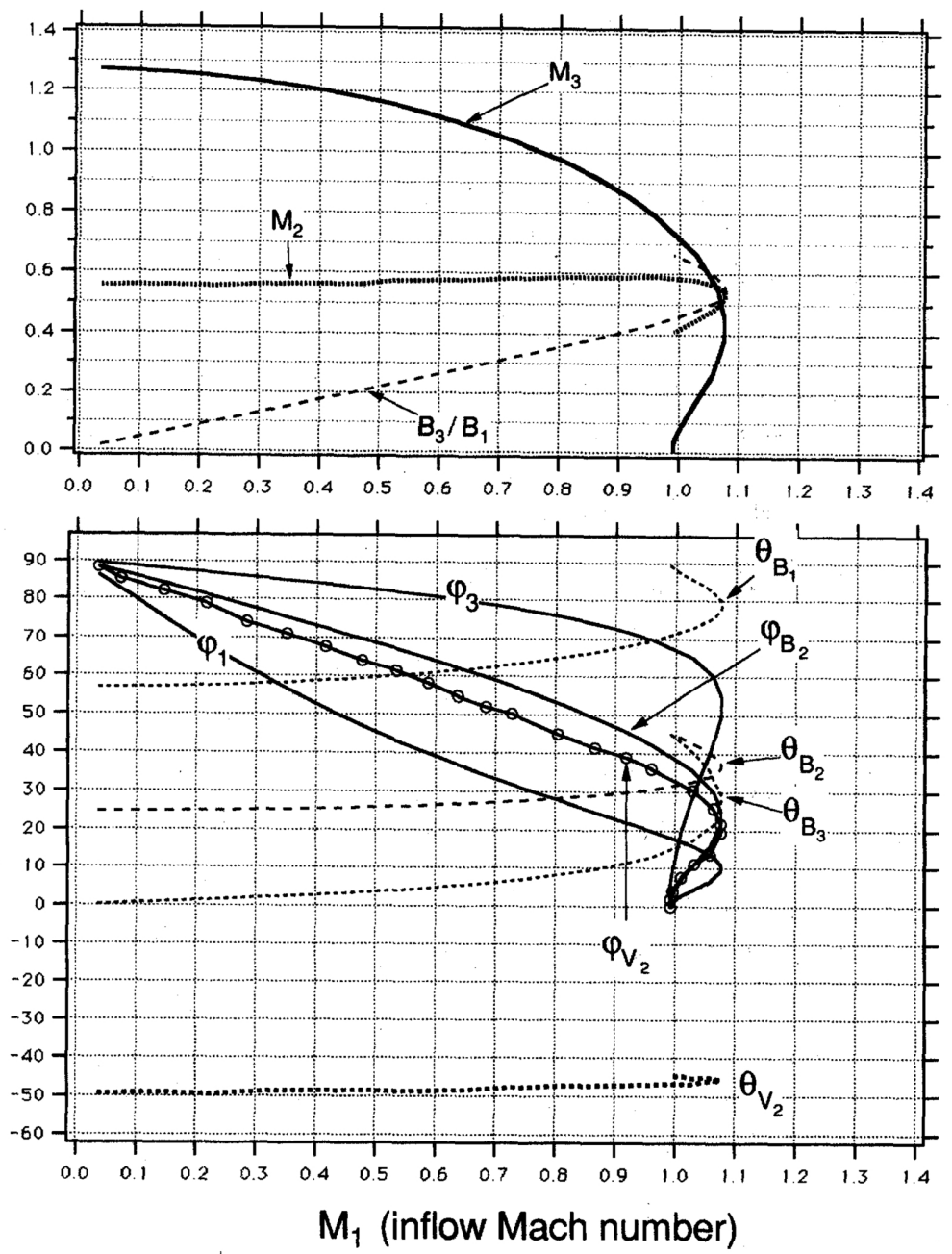

Fig. 2b. Dependence of reconnection parameters on the inflow Alfvén Mach number $M_{1}$ for the case of upstream $\beta_{1}=1$.

rotational discontinuity $R_{a}$. Since $\theta_{V_{2}}<0, V_{z}$ is negative in the region between $R_{a}$ and $S_{a}$ (and also between $R_{b}^{\prime}$ and $S_{b}^{\prime}$ ). In the regions between $R_{b}$ and $S_{b}$, and $R_{a}^{\prime}$ and $S_{a}^{\prime}$, on the other hand, $V_{z}$ is positive. Therefore, the distribution of $V_{z}$ around the neutral line $\mathbf{N}$ has a quadrupole-type structure. Downstream the slow shocks $\left(\mathrm{S}_{\mathrm{a}}, \mathrm{S}_{\mathrm{b}}, \mathrm{S}_{\mathrm{a}}^{\prime}\right.$, and $\left.\mathrm{S}_{\mathrm{b}}^{\prime}\right)$ the plasma flow is deflected in the $\pm y$ directions. These plasma jets in the $\pm y$ direction downstream of the slow shocks are typical of the reconnection geometry.

Above certain values of $M_{1}$ ( $>0.8$ for $\beta_{1}=0$ and $>1.0$ for $\beta_{1}=1$ ), the solution becomes nonunique: For the same $M_{1}$ there are two solutions with higher and lower $M_{3}$. This finding is similar to what Hameiri found in his model. However, as seen in Fig. 2a and 2b, the attack angle of the rotational discontinuity against the upstream flow $\left(M_{1}\right), \varphi_{1}$, becomes $\lesssim 30^{\circ}$ for $M_{1} \gtrsim 1$. For the realistic reconnection geometry, we may require that $\varphi_{1}>45^{\circ}$, so that we should limit the discussion to the solutions with $M_{1} \lesssim 0.5$. For low to moderate $M_{1}(0-0.5)$, the value range of the 
angle of the original magnetic fields, $\theta_{B_{1}}$, is quite limited: For $\beta=0$ (Fig. 2a), $55^{\circ}<\theta_{B_{1}}<58^{\circ}$, and for $\beta=1$ (Fig. 2b), $56^{\circ}<\theta_{B_{1}}<60^{\circ}$. Outside the above range of $\theta_{B_{1}}$, solutions of the new class do not exist.

\section{Summary and Comment}

We have described a new class of exact solutions for the three-dimensional reconnection process, which occurs between oblique original magnetic fields. These solutions are not compressible counterparts of the Sonnerup's incompressible solutions, since the new solutions do not connect to the anti-parallel geometry $\left(\theta_{B_{1}} \rightarrow 0\right)$. For the pure anti-parallel geometry, HAMEIRI (1979) found a class of exact solutions for compressible reconnection problem. Although our solutions are similar to his in that both solutions have three-dimensional geometry and consist of slow shocks and rotational discontinuities, our solutions have different symmetry property and form an independent class of exact solutions.

The new solution for the three-dimensional reconnection predicts the existence of plasma jets along the neutral line $\left( \pm V_{z}\right)$ in the regions between the rotational discontinuities and the slow shocks; in addition to the usual plasma jets in the perpendicular direction to the neutral line $\left( \pm V_{y}\right)$ in the downstream regions of the slow shocks. Since our solution has well-behaved velocity fields without any singularity, it provides a counter example against the suggestion by LAU and FINN (1990), and PRIEST and FORBES (1992), that three-dimensional reconnection regions should have velocity singularities. Note that in these previous studies the magnetic field component parallel to the neutral line, $B_{z}$, is assumed to be uniform in the entire reconnection region. We suggest that this assumption is too restrictive and leads these authors to singular (possibly unphysical) solution. In our solution, $B_{z}$ does change at shocks and discontinuities. However, since what we have obtained is mathematically a particular solution but not a general solution, we need further studies to prove or disprove the existence of the velocity singularities in the three-dimensional reconnection region.

We thank Drs. A. Nishida, K. Maezawa, T. Hada, and B. U. Ö. Sonnerup for their valuable discussions. The Editor also thanks K. Maezawa for his assistance in evaluating this paper.

\section{REFERENCES}

GREENE, J. M., Geometrical properties of three-dimensional reconnecting magnetic fields with nulls, J. Geophys. Res., 93, 8583-8590, 1988.

Hameiri, E., Compressible magnetic-field reconnection, J. Plasma Phys., 22, 245-256, 1979.

Hesse, M. and K. Schindler, A theoretical foundation of general magnetic reconnection, J. Geophys. Res., 93, $5559-5567,1988$.

LAU, Y.-T. and J. M. FinN, Three-dimensional kinematic reconnection in the presence of field nulls and closed field lines, Astrophys. J., 350, 672-691, 1990.

Priest, E. R. and T. G. Forbes, Magnetic flipping: Reconnection in three dimensions without null points, $J$. Geophys. Res., 97, 1521-1531, 1992.

Schindler, K., M. Hesse, and J. BiRN, General magnetic reconnection, parallel electric fields, and helicity, J. Geophys. Res., 93, 5547-5557, 1988.

SonneruP, B. U. Ö., Magnetic-field re-connexion in a highly conducting incompressible fluid, J. Plasma Phys., 4, 161-174, 1970.

Wright, A. N. and M. A. BERGER, The effect of reconnection upon the linkage and interior structure of magnetic flux tubes, J. Geophys. Res., 94, 1295-1302, 1989. 